\title{
A Novel Method for Fault Diagnosis of Planetary Gearbox
}

\author{
Huiling Liu* and Jianguo Zhang \\ School of Mechanical Engineering, Jinzhong University, Jinzhong, 030619, China \\ ${ }^{*}$ Corresponding author
}

\begin{abstract}
The transmission path of planetary gearbox is complex and the fault signal can easily be submerged in the noise signal, so a novel method for fault diagnosis of planetary gearboxes was proposed in the paper, which combined the simulation modeling with the sensor signal processing. Through the dynamic modeling and simulation of the planetary gearbox, it was found that the frequency domain characteristic parameters of the simulation signal can identify different types of sun gear failures. The practical signals were analyzed by the methods of the local wave decomposition, approximate entropy, rough set theory. The minimal fault characteristic parameter set of planetary gearboxes was obtained. The results show that the minimal fault feature parameter set can diagnose different fault types quickly and efficiently with the accuracy of up to $95 \%$.
\end{abstract}

Keywords-planetary gearbox; fault diagnosis; modeling; empirical mode decomposition; rough set

\section{INTRODUCTION}

Planetary gearbox, as the key components of major equipment such as wind power generation, fully mechanized mining equipment and helicopters, usually consist of a sun gear, a ring gear, a planet carrier and a plurality of planet gears. Planetary gears not only need to rotate, but also revolve around the sun gear. This special physical structure determines its own characteristics and difficulties in fault diagnosis. Specifically, the transmission path in the planetary gearbox is complicated, the multi-tooth meshing causes the vibration response to be sometimes enhanced and sometimes suppressed, the vibration response component is complex and has non-stationary characteristics, and the fault signal is easily submerged in an incoherent signal such as noise. In view of its important role in the mechanical transmission system, the research on the method of fault diagnosis for planetary gearbox has positive significance in guaranteeing the reliable operation of mechanical transmission system and reducing the economic cost caused by maintenance and shutdown.

Domestic and foreign scholars have studied the dynamic characteristics and fault diagnosis of planetary gearboxes. Parker considered the gear meshing phase and the influence of the nonlinear parameters on the dynamic characteristics of the system, established the centralized parameter model of planetary gear drive system to study the nonlinear dynamics[1]. Inalpolat from America proposed the discrete two-dimensional nonlinear time-varying dynamics model of planetary gearboxes [2]. Velex from France considered the planetary-wheel position error synthetically and improved the centralized parameter model of planetary gear system. The influence of planetarywheel position error on planetary quasi-static and dynamic load was also studied [3]. Sun Zhimin established A torsional vibration model formulated by time-varying mesh stiffness and composite gear errors to predict the dynamic characteristics of the encased differential gear train[4]. Cheng Zhe built the lumped parameter dynamical models and the multi-body dynamical models of common damages in planetary gear set[5]. Hossam A. Gabbar from Canada used adaptive feature extraction algorithm to deal with non-stationary fault signals, and then proposed a real-time detection of gearbox fault method[6]. Professor Chu Fulei has carried out some researches on the fault diagnosis of rolling bearings and proposed a wavelet energy spectrum signal processing method which can effectively extract the time and frequency domain characteristics of the impact components in the vibration signal[7]. Professor Chen Jin has done some research in the rolling bearing, which could diagnose quickly whether a certain fault occurred in the rolling bearing with using the constrained independent component analysis method [8]. Liu Zhiliang improved the performance of data-driven methods for fault diagnosis of planetary gearboxes[9].

In this paper, a novel method of fault diagnosis of planetary gearboxes is proposed. The typical damage of gears would be modeled, At the same time a data-driven fault diagnosis system of planetary gearbox based on local wave decomposition, rough set theory and information entropy would be established. The combination of modeling for simulation and practical signal processing could provide the foundation for the reliability design of planetary gearbox to reduce the failure rate of major equipment transmission systems such as wind power generations, fully mechanized mining equipment and helicopters.

\section{Mechanical Modeling For Planetary Gearbox}

\section{A. Structure Characteristics of Planetary Gearbox}

The planetary gearbox has structure characteristics such as compact, small size, large transmission ratio, high efficiency, which is a widely used in the revolving gear transmission systems. $2 \mathrm{~K}-\mathrm{H}$ type is one of the most common planetary gear drives, which consists of a sun gear, a ring gear, a number of planetary gears and a carrier. As long as either one of the sun gear, ring gear, and carrier is fixed, the remaining components can be either input or output. The three-dimensional model is shown in Figure I, and the schematic diagram of the structure is shown in Figure II. 


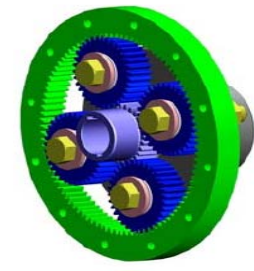

FIGURE I. THREE-DIMENSIONAL MODEL.

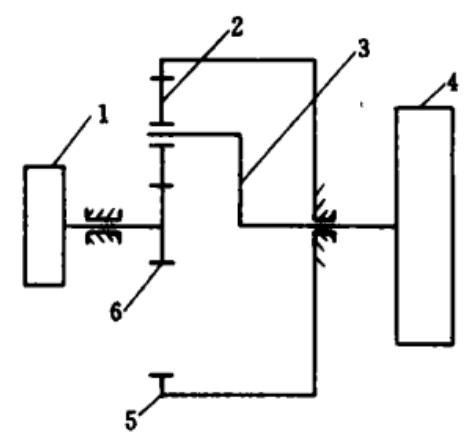

FIGURE II. SCHEMATIC DIAGRAM OF 2K-H PLANETARY GEAR TRAIN. (1.INPUT 2.PLANETARY GEAR 3.CARRIER 4.OUTPUT 5. RING GEAR 6.SUN GEAR)

For $2 \mathrm{~K}-\mathrm{H}$ planetary gear train, it is assumed that the input shaft rotates the sun gear as an input and the carrier drives the load as an output. $Z_{\mathrm{s}}$ represents the number of teeth of the sun gear, $z_{p}$ is the number of teeth of the planet gear, $z_{r}$ is the number of teeth of the ring gear, then the gear ratio $i_{s c}$ of a $2 \mathrm{~K}$ $\mathrm{H}$ planetary gear train could be calculated by (1):

$$
i_{s c}=1+\frac{z_{r}}{z_{s}}
$$

\section{B. Modeling and Simulation}

The centralized parameter model is the most common form of modeling[5], simplifying the various components of a planetary gear train into a concentrated mass, simplifying the connection between components and between the components and the foundation as a spring, thereby equating the planetary gear train with the typical multiple freedom Degree spring mass vibration system, this paper established a pure torsional vibration model of planetary gear train. Before establishing a purely torsional dynamic model of a $2 \mathrm{~K}-\mathrm{H}$ planetary gear train, the following assumptions are made first: (1) All components are free from axial forces and all their vibration vectors lie in a plane perpendicular to the axis; (2) Planetary gears are circumferentially arranged, and the dynamics parameters, quality attributes and meshing process of all the planetary gears are exactly the same; (3) simplify the cage and tie rod as a rigid body; (4) ignore the center lateral displacement of the planetary gears and ring gear; (5) Regardless of the uneven load on the planetary gears. Based on the above assumptions, a purely torsional vibration model of $2 \mathrm{~K}-\mathrm{H}$ planetary gear train can be obtained, as shown in Figure III.
The gear-meshing forces in Figure III between the sun gear and planetary gear, the planetary gear and ring gear approximate as elastic meshing force and viscous engagement force, $\mathrm{K}_{\mathrm{spi}}$ is meshing stiffness of the sun gear and the i-th planetary gear, $\mathrm{K}_{\mathrm{rpi}}$ is meshing stiffness of the ring gear and the i-th planetary gear. $\mathrm{C}_{\mathrm{spi}}, \mathrm{C}_{\mathrm{rpi}}$ respectively represent the meshing damping along the line of action of the sun gear and the i-th planetary gear, the i-th planetary gear and the ring gear. $\theta_{\mathrm{s}}, \theta_{\mathrm{pi}}$ and $\theta_{\mathrm{c}}$ denote the rotation angles of sun gear, the i-th planetary gear, cage. TD, TL, respectively are driving torque and load torque.

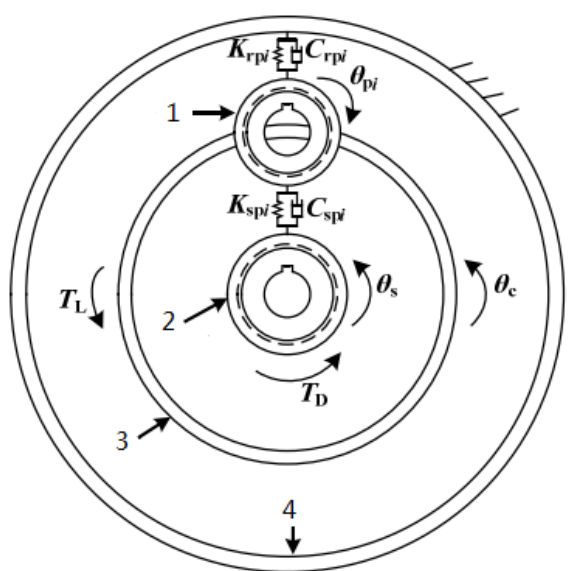

FIGURE III. PURELY TORSIONAL VIBRATION MODEL OF THE PLANETARY GEAR TRAIN.(1. PLANETARY GEAR 2. SUN GEAR 3. CARRIER 4. RING GEAR)

Planetary gear train in operation often fails due to poor manufacturing and poor operation and maintenance and other causes of damage, and the type of damage also with the gear material, heat treatment process, operating conditions and other factors vary. In this paper, we mainly study the types of faults prone to the sun gear, such as cracks, pitting and broken teeth. ADAMS software is used to model the dynamics of the planetary gear train failure mode. The virtual sensors are placed in the vertical direction of the ring gear. It is found that the influence of different modes of sun gear failure on the simulation signals in the frequency domain is quite different, mainly because the frequency distributions are different. The conclusion could be got that the damage can be detected and the damage pattern can be identified by constructing the frequency domain feature.

\section{Practical Signal Processing Method}

\section{A. Empirical Mode Decomposition}

The traditional signal processing methods in core of Fourier are not suitable for processing the non-stationary signal with low signal noise ratio and strong interference. Empirical Mode Decomposition(EMD) is self-adaptive and can overcome the defects of traditional signal processing method in processing non-stationary and nonlinear signal, fundamentally eliminate the influence of human factors and achieve better results[10]. At the same time the frequency domain characteristic parameters could be got, which is consistent with Part II. 
EMD[11,12,13] decompose the signal on the time scales. It can decompose the complex signal into a finite number of intrinsic mode functions (IMF), and each intrinsic mode function can express the natural mode of vibration in the original signal and reflect the local signal characteristics well. In the signal decomposition based on EMD, according to different frequency, the intrinsic mode functions with different fluctuation characteristics and trends are decomposed from the original signal in the sequence and every IMF is stationary signal, which converts the non-stationary signal into smooth successfully.

In the extract method of energy characteristics based on EMD, every decomposed IMF will be processed further to extract more effective feature parameters. The main steps are as follows:

(a) Decomposing every sample based on EMD to get intrinsic mode functions with different frequency.

(b) Computing the total energy of the intrinsic mode function.

$$
E_{c k}=\left|\int C_{k}(t)\right|^{2} d t=\sum_{i=1}^{n}\left|C_{k j}\right|^{2}
$$

Where, $C_{k j}(j=1,2, \mathrm{~L})$ is the signal amplitude of $\mathrm{j}$ discrete point on $\mathrm{k}$ layer.

(c) Constructing feature vectors and normalization.

$$
\begin{gathered}
T=\left[E_{c 1}, E_{c 2}, \mathrm{~L}, E_{c n}\right] \\
E=\left(\sum_{k=1}^{n}\left|E_{c k}\right|^{2}\right)^{1 / 2} \\
T^{\prime}=\left[E_{c 1} / E, E_{c 2} / E, \mathrm{~L}, E_{c n} / E\right]
\end{gathered}
$$

Where $T^{\prime}$ is the normalized feature vector.

\section{B. Approximate Entropy}

In 1991, Steven M. Pincus proposed using approximate entropy to measure the complexity of time series, mainly to measure the probability of generating new modes in the vibration signal[14]. The more complex the signal, the larger the approximate entropy will be. Assumed that the original data sequence contain $N$ values $\{u(i), i=1,2, \mathrm{~L}, N\},\{u(i)\}$ is the definite value $i$. Assuming that the dimension of the model is $m$, the similar capacity is $r$, the approximate entropy calculation procedure is as follows: (a) $M$ dimension vectors $X(i)$ are composed of the sequence $\{u(i)\}$, expressed as follows:

$$
X(i)=[u(i), u(i+1), \mathrm{L}, u(i+m+1)]
$$

(b) Calculate the distance between the vector $X(i)$ and $X(j)$ by the following formula:

$$
d[X(i), X(j)]=\max _{k=0: m-1}|u(i+k)-u(j+k)|
$$

Where, $d[X(i), X(j)]$ denotes the distance between two vectors.

(c) Given the threshold $r(r>0)$, choose different values to calculate the number $d[X(i), X(j)]<r$, while calculating the ratio that can be written as:

$$
C_{i}^{m}(r)=\{d[X(i), X(j)]<r\} /(N-m+1)
$$

(d) Take the logarithm of $C_{i}^{m}(r)$, and then take the average of the logarithm, recorded as $\Phi^{m}(r)$ :

$$
\Phi^{m}(r)=\frac{1}{N-m+1} \sum_{i=1}^{N-m+1} \ln C_{i}^{m}(r)
$$

(e) Add 1 to the dimension, that is $m+1$, then repeat the above four steps to get $\Phi^{m+1}(r)$.

(f) Theoretically, the approximate entropy can be calculated by the following formula:

$$
A_{p}^{E n}(m, r)=\lim _{N \rightarrow \infty}\left[\Phi^{m}(r)-\Phi^{m+1}(r)\right]
$$

In fact, the value of $N$ is finite, it is impossible to infinity, so approximate entropy can be approximated by the following formula:

$$
A_{p}^{E n}(m, r, N)=\Phi^{m}(r)-\Phi^{m+1}(r)
$$

In this paper, the practical signals of these kinds of faults such as cracks, pitting and broken teeth of the sun gear are analyzed by EMD respectively. Energy characteristics and approximate Entropy parameters are obtained based on the first six IMF components, 12 parameters totally. There are many characteristic parameters, so it is not easy to quickly diagnose 
the fault pattern. Next rough set theory will be used to optimize the characteristic parameter set.

\section{ROUGH SET THEORY}

\section{A. Basic Concepts}

The notion of an information system is described in the works of Pawlak and Yasdi $[15,16]$. A summary is included for completeness here.

An information system $S=\left(U, \Omega, V_{q}, f_{q}\right)$ consists of: $U$-- a nonempty, finite set called the universe; $\Omega$-- a nonempty, finite set of attributes; $\Omega=C \cup D$, where $C$ is a finite set of condition attributes and $D$ is a finite set of decision attributes; For each $q \in \Omega, V_{q}$ is called the domain of $q ; f_{q}{ }^{--}$an information function $f_{q}: U \rightarrow V_{q}$.

If $S=\left(U, \Omega, V_{q}, f_{q}\right)$ is a decision table, $B \subseteq \Omega$ and $X \subseteq U$ then B-lower and B-upper approximation of $X$ is defined respectively as follows:

$$
\underline{B} X=\cup\{Y \in U / B \mid Y \subseteq X\}
$$

$$
\bar{B} X=\cup\{Y \in U / B \mid Y \cap X \neq \phi\}
$$

If $C \subseteq \Omega$ and $D \subseteq \Omega, P_{C}(D)$ is $C$ Positive region of $D$ :

$$
\operatorname{POS}_{C}(D)=\underset{X \in U / D}{\mathrm{U}} \underline{C X}
$$

If $S \subseteq C$, only when $S$ is $D$ independent subset of $C$, and $\operatorname{POS}_{S}(D)=\operatorname{POS}_{C}(D), S$ is $D$ reduction of $C$.

\section{B. Key Technical Issues}

Rough set theory mainly involves three main problems: continuous attribute discretization, conditional attribute reduction, value reduction [17-19]. This paper proposes a global dynamic optimization discretization algorithm based on improved Naive Scaler algorithm. Firstly, the advantages and disadvantages of Naive Scaler algorithm are analyzed. Based on the original algorithm, the calculation process is improved so that the algorithm can get all the breakpoints that guarantee the indefinable relationship. Then break points are gradually increased by evenly dividing the sample set The method selects the set of breakpoints dynamically from the candidate set to ensure that the number of breakpoints is the least under the condition of the same classification ability of the information system. This paper proposes a attribute reduction algorithm based on conditional equivalence classes. The basic idea is: first, we get the set of kernel attributes by the definition of kernel attributes, and then for the attributes of the core attributes that cannot be correctly classified into decision classes, To find the attributes that can distinguish this class from the rest of the conditional attributes to get all the minimal attribute reduction sets. A greedy algorithm based on decision matrix is proposed to reduce the value. The main idea is to establish a binary decision table for each sample according to the idea of decision matrix. The greedy algorithm is used to reduce the decision-making rules.

Rough set theory is used to attribute reduction of planetary gearbox fault feature parameter set built in part III. The fault feature set which is composed of 6 characteristic parameters is obtained. At the same time, fault type decision rules are extracted, and the diagnostic accuracy rate reaches above $95 \%$.

\section{CONCLUSION}

In this paper, the new method of fault diagnosis of planetary gearboxes is proposed. Through the dynamic modeling and simulation of planetary gearboxes, it is found that the influence of different types of sun gear faults on the frequency domain of the simulation signals is quite different. The practical signals are analyzed by combining the local wave decomposition, approximate entropy, rough set theory, the minimal fault characteristic parameter set of planetary gearboxes is obtained. Next, the key technical issues such as dynamic modeling of planetary gearboxes, local wave decomposition, rough set theory and the configuration of sensor measurement points need to be further studied to obtain a more sensitive and stable fault characteristic parameter set.

\section{ACKNOWLEDGMENT}

The paper is supported by Applied Basic Research Programs of Shanxi Province (No.201701D221132), Bo Shi Ji Jin Programs of Jinzhong University (bsjj2015205).

\section{REFERENCES}

[1] K. Ambarisha, R. G. Parker, "Nonlinear dynalnics of planetary gears using analytical and finite element models," Journal of Sound and Vibration,Vol. 302, No.3, 2014, pp. 577-595.

[2] M. Inalpolat, A. Kahraman, "A dynamic model topredict modulation sidebands of a planetary gear set having manufacturing errors," Journal of Sound and Vibration, Vol. 329, 2014, PP. 371-393.

[3] X. Gu, P. Velex, "A dynamic model to study the influence of planet position errors in planetary gears," Journal of Vibration and Acoustics, Vol. 331, No. 20, 2012, pp. 4554-4574.

[4] Sun Zhimin, Shen Yunwen, Li Suyou, " A Study on Torsional Vibrations in an Encased Differential Gear Train," Journal of Aerospace Power, Vol. 16,No.2, 2001, pp. 163-166.

[5] Cheng Zhe, Theory and Method on Damage Modeling and Prognostics for Planetary Gear Set of Helicopter Transmission System. Changsha, CHN: Graduate School of National University of Defense Technology, 2011.

[6] S. Hussain, H. A. Gabbar, "A novel method for real time gear fault detection based on pulse shape analysis," Mechanical System and Signal Processing, Vol. 25, No.4, 2011, pp. 1287-1298.

[7] Ming Anbo, Chu Fulei, Zhang Wei, "Feature Extracting Method in the Rolling Element Bearing Fault Diagnosis: Spectrum Auto-correlation," Journal of Mechanical Engineering, Vol. 48, No.19, 2012, pp. 65-71.

[8] Zhou Yu, Chen Jin, Dong Guangming, etal., "Fault diagnosis of rolling element bearing based on cyclic bispectrum,"Journal of Vibration and Shock, Vol.31, No.9, 2012, pp. 78-81.

[9] Liu Zhiliang, Data-Driven Fault Diagnosis For Planetary Gearboxes. 
Chengdu, CHN: University of Electronic Science and Technology of China.

[10] Norden E. Huang, Zheng Shen, "The empirical mode decomposition and the Hilbert spectrum for nonlinear and The empirical mode decomposition and the Hilbert spectrum for nonlinear and non-stationary time series analysis," Proceeding of Royal Society, London, Vol. 454(A) 1998, pp. 903-995.

[11] Lihong Qiao, Sisi Chen, "Bi-dimensional Empirical Mode Decomposition And Developed 2d Hilbert Spectrum Characters For The Segmentation Of Texture," AISS, Vol. 4, No. 9, 2012, pp. $319 \sim 327$.

[12] Tang Hong-bin, Wu Yun-xin, Liao Zhi-qi, "Fault Diagnosis of Hydraulic Pump Bearing Based on EMD and Envelope Spectrum," JDCTA, Vol. 5, No. 12, 2014, pp. $28 \sim 34$.

[13] Xiaolei Zhang, Yi Shen, Naizhang Feng, Zhenhua Wang, "An Empirical Mode Decomposition Signal Denoising Method Based on Block Energy," IJACT, Vol. 4, No. 4, 2012, pp. $226 \sim 234$.

[14] A.J.C.Sharkey, G.O.Chandroth, N.E.Sharkey, "A Multi-Net System for the Fault Diagnosis of a Diesel Engine,” Neural Comput \& Applic, No.9, 2000, pp. 152-160.

[15] Z. Pawlak, "Rough sets,"Computer and Information Science, Vol.11, No.2, 1982, pp. 341-356.

[16] R.Yasdi, "Combining Rough Sets Learning and Neural Learning method to deal with uncertain and imprecise information," Neurocomputing7, 1995, pp. 61-84.

[17] $\mathrm{Hu}$ XiaoHua, Cercone N, "Learning in relational databases:a rough set approach," Computational Intelligence, Vol.11, No.2, 1995, pp. 323337.

[18] Shen Lixiang, Francis E H T, Qu Liangsheng, et al., "Fault diagnosis using rough sets theory," Computers in Industry, Vol.43, No.2, 2000, pp. 61-72.

[19] Czogala E, Mrozek A, Pawlak Z, "The idea of a rough fuzzy controller and its application to the stabilization of a pendulum-car system," Fuzzy sets and systems, Vol.72, No. 1, 1995, pp. 61-73. 